\title{
Resonant flights and transient superdiffusion in a time-periodic, two-dimensional flow
}

\author{
T.H. Solomon*, Andrew T. Lee ${ }^{1}$, M.A. Fogleman ${ }^{2}$ \\ Department of Physics, Bucknell University, Lewisburg, PA 17837, USA
}

Received 27 November 2000; received in revised form 27 April 2001; accepted 1 May 2001

Communicated by E. Ott

\begin{abstract}
Enhanced, passive transport is studied numerically in an oscillating vortex chain with stress-free boundary conditions. The long-range transport is found to be diffusive in the long-time limit with an effective diffusion coefficient $D^{*}$ that peaks dramatically in the vicinity of a few, well-defined resonant frequencies. Superdiffusive transients are also observed for frequencies near these resonant frequencies, with the duration of the transients diverging at the resonant frequencies. Standard analytical techniques based on the Melnikov approximation and on lobe dynamics fail to explain the behavior in the vicinity of these resonant peaks. An alternate explanation is provided, based on flights that have power-law scaling up to a maximum length that also diverges at the resonant frequencies. The long flights for frequencies near the resonant peaks occur because tracers in a lobe return (after an integer number of oscillation periods) to almost precisely the same location in the lobe of another vortex. These periodic orbits correspond to the formation - only at the resonant frequencies — of "tangle islands" within the chaotic region. (C) 2001 Elsevier Science B.V. All rights reserved.
\end{abstract}

PACS: 05.40.Fb; 47.52.+j; 94.10.Lf; 05.45.Pq

Keywords: Lagrangian chaos; Lévy flights; Superdiffusion

\section{Introduction}

Theories of deterministic chaos have been shown to have significant applications in studies of fluid mixing [1-4]. Simple, laminar fluid flows can advect passive tracers in surprisingly complicated trajectories

\footnotetext{
${ }^{*}$ Corresponding author. Tel.: +1-570-577-1348; fax: +1-570-577-3153.

E-mail addresses: tsolomon@bucknell.edu (T.H. Solomon), atlee@ chaos.ph.utexas.edu (A.T. Lee), fogleman@mae.cornell.edu (M.A. Fogleman).

${ }^{1}$ Current address: Center for Nonlinear Dynamics, University of Texas at Austin, Austin, TX 78712, USA.

${ }^{2}$ Current address: Department of Mechanical and Aerospace Engineering, Cornell University, Ithaca, NY 14850, USA.
}

that separate exponentially in time ("sensitive dependence on initial conditions"). Lagrangian chaos - as this process is called - has dramatic effects on the efficiency of mixing in these flows. In fact, Lagrangian chaos has already found applications in chemical mixing processes for viscous flows where turbulent mixing would be too expensive energetically.

Studies of Lagrangian chaos have been extended to investigate long-range transport - mixing of impurities over distances much longer than typical length scales of the flow [5-7]. Chaotic motion of the tracers replaces Brownian motion as the dominant mechanism for mixing, resulting in significant enhancements in long-range transport beyond that due to molecular 
diffusion. Long-range transport is typically analyzed by studying the growth of the variance $\left\langle x^{2}\right\rangle$ of a distribution of passive tracers as a function of time. If the variance grows as a power law in time $\left\langle x^{2}\right\rangle \sim t^{\gamma}$ with $\gamma=1.0$, then the process is referred to as normal, enhanced diffusion. On the other hand, transport with $\gamma<1$ or $\gamma>1$ correspond to what are called "sub-diffusion" or "superdiffusion," respectively. In the long-time limit, subdiffusion is not allowed for incompressible velocity fields in the absence of molecular diffusion [8].

In cases where the transport is normal, enhanced diffusion, two standard theoretical approaches are typically used to predict enhanced diffusion coefficients $D^{*}$ : Melnikov analysis and lobe dynamics. Both of these approaches are based on a determination of the flux of tracers between adjacent vortices during one oscillation period.

Recent theories indicate that superdiffusive transport is associated with tracers whose trajectories can be characterized as "Lévy flights" [9-11], where tracers can jump very long distances (relative to typical length scales in the flow) between regions in which they are temporarily confined. The connection between superdiffusive transport and Lévy flights has been verified experimentally in two-dimensional (2D), quasi-geostrophic (planetary-type) fluid flows [12,13].

Theories of deterministic chaos have been instrumental in understanding how Lévy flights can arise in fluid mixing problems. Typically, a flow is divided into ordered and chaotic regions, categorized by the trajectories of tracers moving in the regions. The regions are separated by "KAM barriers" across which no tracer can cross. Tracers in the chaotic regions can come arbitrarily close to a KAM barrier, though, in which case they "stick" temporarily, mimicking the behavior of ordered trajectories in the region bounded by that barrier $[14,15]$. If some of the ordered regions correspond to unbounded motion in which tracers travel long distances in short times, then sticking to the KAM barriers surrounding these regions will result in long flights [16].

In this paper, we present numerical studies of resonant superdiffusion in a time-periodic, 2D flow consisting of an oscillating vortex chain. Mixing in this flow is typically "normal" $(\gamma=1.0)$ enhanced diffusion $[17,18]$ with an enhanced diffusion coefficient $D^{*}$ that depends linearly on oscillation amplitude (if small) and non-trivially on the frequency. At certain precise frequencies, though, the transport is superdiffusive, consistent with previous theoretical studies of this system [19]. Near — but not at — these resonant frequencies, we find that there is still a significant transient regime of superdiffusive behavior, after which the system settles to normal diffusion with a $D^{*}$ that is significantly enhanced by the transient superdiffusion. Standard approaches based on Melnikov and lobe analyses fail to explain not only the transient superdiffusion but also the long-term $D^{*}$ for frequencies near the resonant peaks.

We show that near the resonant frequencies, tracer trajectories show flights with scaling properties similar to a Lévy distribution for small length flights. These flights dominate the transport statistics, explaining the failure of the Melnikov and lobe analyses, which consider behavior only on timescales of one oscillation period and distances of one vortex width. At the resonant frequencies, periodic orbits are found within the lobes - along with very small "tangle islands" [20] - that explain the presence of flights at these frequencies. These periodic orbits disappear for frequencies away from the resonance peaks.

In Section 2, we discuss theories of Lagrangian chaos and long-range transport, and present the model flow. The results of numerical simulations of this flow are presented in Section 3. Different approaches are used to explain the results of the simulations in Section 4, and a summary is presented in Section 5.

\section{Background and model flow}

\subsection{Oscillating vortex chain flow}

Whereas Lagrangian chaos can occur in threedimensional (3D) time-independent flows [21,22], it is possible in 2D flows only if the flow is time-dependent. The flow studied in this paper is a particularly simple time-dependent flow: a 2D, time-periodic flow composed of an oscillating vortex chain which was first proposed $[6,23]$ as a simple model of time-periodic 


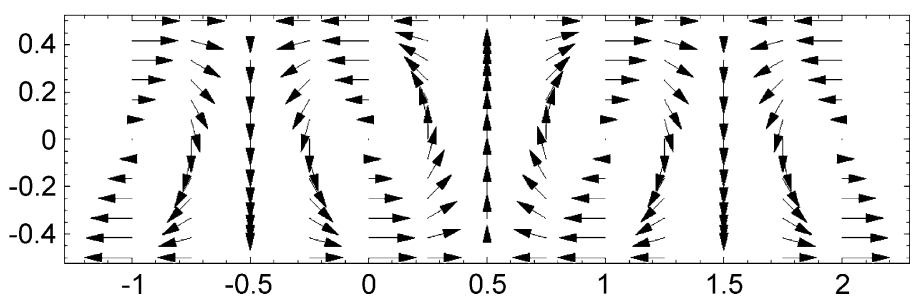

Fig. 1. Velocity field for oscillating vortex chain.

Rayleigh-Bénard (RB) convection [24]. The equations describing this flow are as follows:

$\dot{x}=-a \frac{\lambda}{2 d} \cos \left(\frac{2 \pi(x+B \sin \omega t)}{\lambda}\right) \sin \left(\frac{\pi y}{d}\right)$,

$\dot{y}=a \sin \left(\frac{2 \pi(x+B \sin \omega t)}{\lambda}\right) \cos \left(\frac{\pi y}{d}\right)$.

In these equations, $a$ is the maximum speed, $d$ the vortex height, $\lambda$ the wavelength of the vortex chain, and $B$ the amplitude of the lateral oscillation of the vortex chain. The flow satisfies the incompressibility condition $\vec{\nabla} \cdot \vec{v}=0$. A plot of the velocity field at an instant in time is shown in Fig. 1. As the flow evolves in time, the entire velocity field oscillates back and forth in the horizontal $(x)$ direction.

A simplification implicit in this model is the assumption of free-slip boundary conditions, even though most real convective flows have no-slip (rigid) boundary conditions. Despite this simplifying assumption, the model captures important features that are characteristic of Lagrangian chaos in a wide variety of time-periodic, 2D flows. A follow-up to this paper will compare this model to one with no-slip boundary conditions, and compare both with on-going experimental studies.

The first experimental studies of transport in this system utilized time-periodic RB convection [6,23]. Those studies first demonstrated the possibility for Lagrangian chaos in this system and showed the applicability of this model to real flows. Furthermore, the experiments found that the transport could be described as normal, enhanced diffusion where the variance $\left\langle x^{2}\right\rangle$ of a distribution of tracers grows as $t^{\gamma}$ with $\gamma=1$. Specifically, $\left\langle x^{2}\right\rangle=2 D^{*} t$, where $D^{*}$ is the enhanced diffusion coefficient. It was found that
$D^{*}$ grows linearly with amplitude of oscillation (for small amplitudes), although quantitative comparisons between the model and experiment were complicated by weak, secondary, 3D flows and by the fact that the primary instability to periodic time-dependence in $\mathrm{RB}$ convection is not necessarily a pure lateral oscillation (depending on the Prandtl number of the fluid) [24]. Those experiments were followed by theoretical studies that explained the linear dependence on amplitude [7].

More recent experiments utilized magneto-hydrodynamically forced vortex chains, resulting in much more carefully controlled experiments $[17,18]$. The results - which still indicated normal, enhanced diffusion - were analyzed and explained using a theory based on lobe (turnstile) dynamics [5,25,26], which is described in more detail in Section 4. Those experiments also extended the studies to include transport of immiscible impurities.

Recent theoretical studies of this system [19] have begun to address the dependence of the transport on the frequency of oscillation. The result is a very complicated dependence of $D^{*}$ on $f$ with sharp, upward peaks at various frequencies. Also, infinitesimally thin windows of superdiffusive behavior were found in those studies. One of the primary goals of the studies described in this paper is to provide an explanation for these superdiffusive regimes.

\subsection{Lévy flights and superdiffusion}

A Lévy flight (sometimes called a "walk") is defined as a trajectory with jumps with a wide range of lengths $L$. The probability distribution function (PDF) $p(L)$ for a Lévy flight has infinite second moment: $\left\langle L^{2}\right\rangle=\int_{0}^{\infty} L^{2} p(L) \mathrm{d} L=\infty[9,10]$. This condition is 
satisfied if the PDF is a power law $p(L) \sim L^{-\mu}$ with $\mu<3$.

The connection between Lévy flights and superdiffusive transport has been investigated by several theorists [11,27-30]. A comprehensive theory relates the exponent $\gamma$ for the growth of the variance to the statistics of both the flights and the trapping events. We limit our discussion here to the case where the trapping events have durations $T$ with finite second moment: $\left\langle T^{2}\right\rangle \neq \infty$. In this regime, if the flight PDFs can be described as power-law relations, then $\gamma$ is predicted to depend on the decay exponent $\mu$ as follows:

$\gamma= \begin{cases}2, & \mu<2, \\ 4-\mu, & 2<\mu<3, \\ 1, & \mu>3 .\end{cases}$

These predictions assume that the flights are statistically independent, an assumption that is somewhat controversial [31]. ${ }^{3}$ The theories also apply only to the long-time limit, a limit that can be difficult to achieve experimentally. Practically, almost every real system displays normal diffusion in the long-time limit, either because of the effects of molecular diffusion (which are always present) or because the finite size of the system limits the lengths that the flights can achieve. Sub-and superdiffusion are still very relevant experimentally, though, as transient regimes can have durations comparable to or even longer than the relevant time scales of many processes. Transient sub-diffusion has been studied both theoretically [32,38] and experimentally [33,39] in a time-independent, 2D vortex flow. Transient superdiffusive transport has received less attention.

\section{Numerical results}

The model equations (Eqs. (1) and (2)) can be non-dimensionalized by scaling all distances by the vortex width $d$, velocities by $a$, and time by the characteristic advective time $\tau_{\mathrm{adv}}=d / a$; this is the time

\footnotetext{
${ }^{3}$ For a discussion of integrating correlations within the framework of Eq. (3), see [37].
}

to traverse a single vortex at the maximum velocity. (There is no single characteristic circulation time as tracers at different distances from vortex centers take different times to complete a full rotation.) Furthermore, in all of the simulations in this paper, the vortices are assigned unit aspect ratio, so $\lambda=2 d$. In non-dimensional form, Eqs. (1) and (2) can be re-written as

$\dot{x}=-\cos (\pi[x+b \sin \omega t]) \sin (\pi y)$,

$\dot{y}=\sin (\pi[x+b \sin \omega t]) \cos (\pi y)$.

The non-dimensional oscillation amplitude $b=B / d$ is 0.12 for all the simulations.

Eqs. (4) and (5) are integrated using a fourth-order Runge-Kutta technique. Fig. 2 shows simulations of the evolution of 10,000 particles (initially distributed within a single vortex) for frequencies $f=0.095$ and 0.106 (parts a and b, respectively). Qualitatively, the behavior seen in these simulations is similar to that from previous experiments; see, e.g., Fig. 2 from Ref. [17] or Fig. 3 from Ref. [18].

A Poincaré section is shown in Fig. 3. Trajectories are determined for five tracers and plotted once every period of oscillation. The phase space (which is real space here) is characterized by large core regions in the vortex centers in which tracers undergo regular, ordered trajectories. Surrounding these core regions is a chaotic web that wraps around and between the vortices. Whereas tracers in the chaotic web are free to move between vortices (contributing to long-range transport), those within the ordered core regions remain confined to their original vortex.

The variance $\left\langle x^{2}\right\rangle$ of tracer distributions is plotted as a function of time in Fig. 4. Typically, the variance grows linearly with time after a brief transient (a few times the characteristic advection times $\tau_{\mathrm{adv}}$ ); see, e.g., the lower two curves in Fig. 4 ( $f=0.115$ and 0.095). For limited ranges of frequencies, though, the variance grows superdiffusively for small times; see, e.g., the upper dashed curve in Fig. 4 ( $f=0.106)$. On occasion, a brief sub-diffusive transient regime will be apparent before the curve flattens out to diffusive behavior; see the solid curve in Fig. $4(f=0.095)$. 

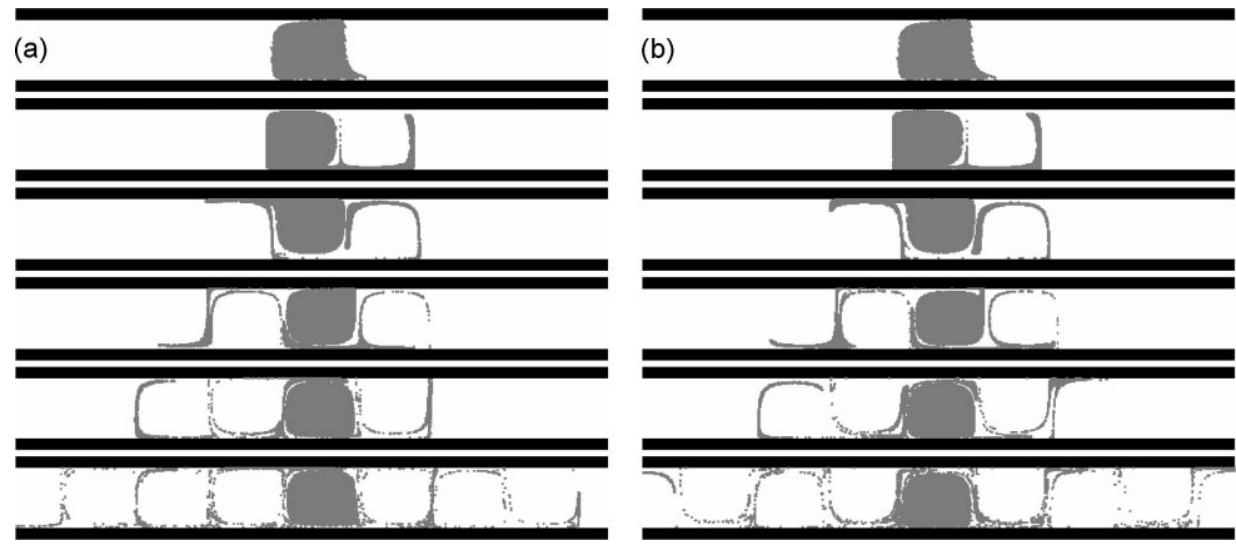

Fig. 2. Sequences showing the evolution of an ensemble of 10,000 tracers, initially distributed in the center vortex: (a) oscillation frequency $f=0.095$; (b) $f=0.106$. From the top, times after beginning of simulation are $t=2,4,6,8,10$ and 20 in non-dimensional units.

Clearly, the long-term transport is very different for these three frequencies. On the other hand, though, the three curves in Fig. 4 are identical for times $t<$ 20; consequently, the dramatic differences between the longer term transport properties for $f=0.095,0.106$ and 0.115 are not simply due to differences in the flux of impurities between vortices during a single oscillation period. This conclusion is also supported qualitatively by Fig. 2; comparing the behavior at $f=$ 0.095 with that for $f=0.106$, there is no significant difference between the two, also indicating that the differences are not explained by a simple flux analysis. These points are discussed further in Sections 4.1 and 4.2.

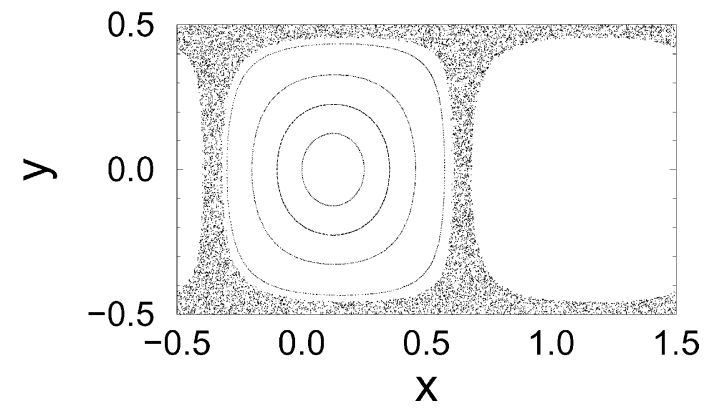

Fig. 3. Poincaré section for $f=0.095$. The trajectories of five tracers (only one of which is in the chaotic region) are determined and plotted once every period of oscillation.
If the transport can be described as "normal" enhanced diffusion $\left(\left\langle x^{2}\right\rangle \sim t^{\gamma}\right.$ with $\left.\gamma=1\right)$, then an enhanced diffusion constant $D^{*}$ can be determined from the slope of a plot of $\left\langle x^{2}\right\rangle$ versus $t$. If the transport is superdiffusive, though, $D^{*}$ is a time-dependent function. As shown by Vulpiani and coworkers [19] the transport is not superdiffusive in the long-time limit except at only certain, precise resonant frequencies, so in general the slope of $\left\langle x^{2}\right\rangle(t)$ can be determined at long times to get an approximation of $D^{*}$. Fig. 5 shows $D^{*}$ (determined from the variance plot for $t>100$ ) plotted as a function of oscillation frequency. Two large resonant peaks are apparent, the first peak occurring at $f=0.106$, and corresponding

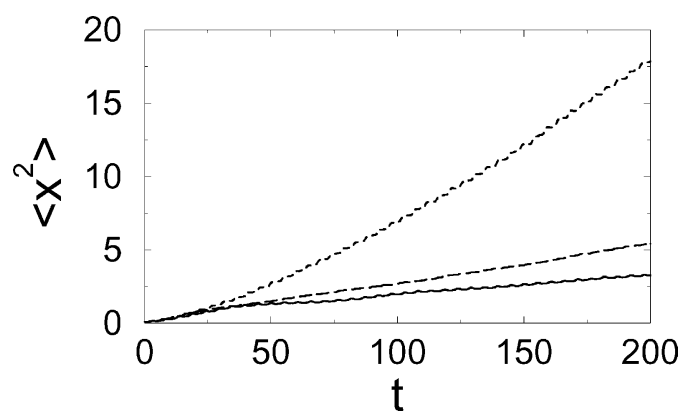

Fig. 4. Growth of variance $\left\langle x^{2}\right\rangle$ of distribution versus nondimensional time $t$. Frequency of oscillation $f=0.095$ (lower, solid line), 0.106 (upper, dashed line) and 0.115 (middle, long-dashed line). 


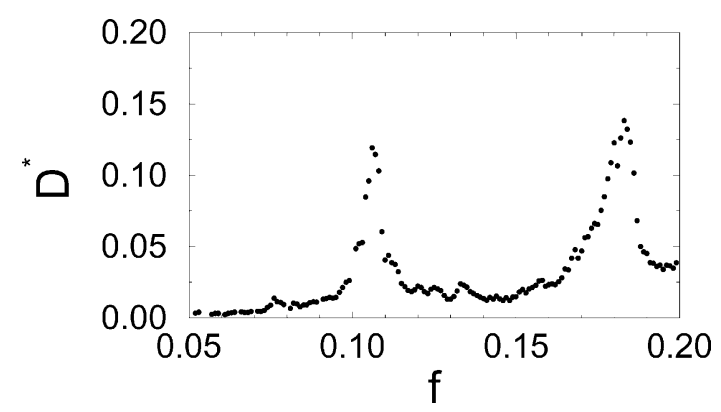

Fig. 5. Enhanced diffusion coefficient $D^{*}$ as a function of frequency $f$ of the oscillation. For all points, $D^{*}$ was determined by taking the slope of the plot of $\left\langle x^{2}\right\rangle$ versus $t$ for $t>100$.

to the top dashed curve in Fig. 4 and the simulation in Fig. 2b.

Fig. 6 shows logarithmic plots of the variance for frequencies close to the first resonance peak at $f=$ 0.106. Even though the transport is superdiffusive in the long-time limit only precisely at the resonant frequency [19], there are transient superdiffusive regimes for frequencies near the resonant frequency, with the duration of these transient regimes decreasing the
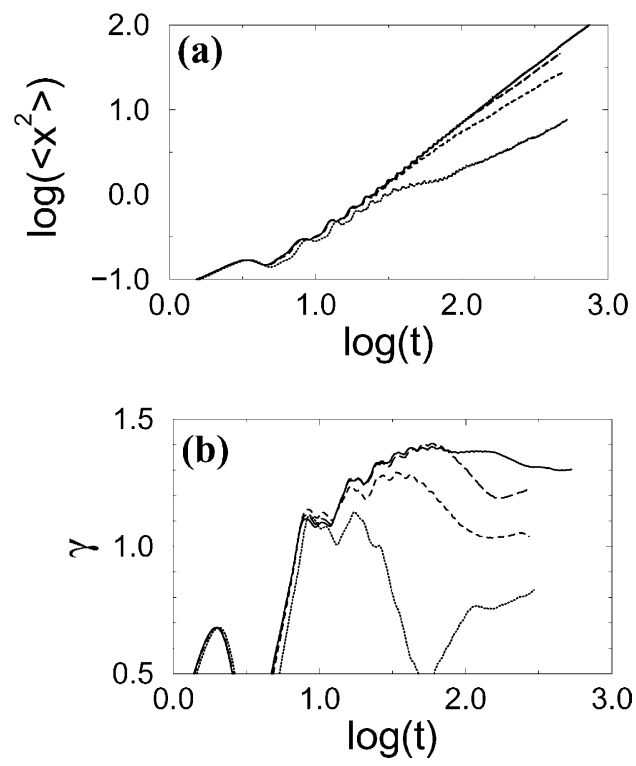

Fig. 6. Growth of variance $\left\langle x^{2}\right\rangle$ versus time $t$; from top, $f=0.106$ (solid curve), $f=0.105$ (long-dashed curve), $f=0.103$ (dashed curve), and $f=0.095$ (dotted curve): (a) logarithmic plot; (b) slope of plots from (a), revealing growth exponent $\gamma$ versus time. farther $f$ is from the resonant frequency. ${ }^{4}$ The transient superdiffusion is followed by normal diffusion in these cases. For frequencies outside the peak regions in Fig. 5, the superdiffusive transients disappear, and the transport is normal $(\gamma=1)$ throughout, with the exception of a few situations (such as at $f=0.095$, bottom dotted curves in Fig. 6), where there is a transient sub-diffusive regime.

Numerical inaccuracy could affect interpretation of the results from Fig. 6. Conceptually, numerical errors have a similar effect on the numerical results as the molecular diffusion has on the experimental results. In particular, the addition of either numerical or Brownian noise limits the timescale for superdiffusive growth of the variance, resulting in normal diffusion at long times. We minimize these effects by choosing a time step in the simulations that is small enough such that the results are insensitive to changes in that step. A better indication of the effects of numerical noise on the results can be seen from the data in Fig. 6 itself. Since the superdiffusive regime at the resonance peak ( $f=0.106)$ is maintained up until at least $t=200$ $(\log (t)=2.3)$, it can be seen that the drop-off in $\gamma$ at earlier times for other frequencies is not due to numerical noise.

\section{Analysis}

The central questions are as follows: (1) What is the cause of the resonance peaks in $D^{*}$ as a function of $f$ (Fig. 5)? (2) Why is the transport superdiffusive at short times in the vicinity of these peaks, and why is this superdiffusive transport of limited duration in these cases?

Commonly, long-range chaotic transport is analyzed theoretically either by looking at "lobes" (or "turnstiles") that are responsible for interchange of tracers between adjacent vortices, or by calculating the Melnikov function for the system. We will examine both of these local approaches to transport in the context of the results presented above.

\footnotetext{
${ }^{4}$ Calculation of $D^{*}$ in the long-time limit is less accurate near the peaks of the resonance curve, since the duration of the transient superdiffusive regime diverges at the resonances.
} 


\subsection{Melnikov analysis}

Transport in Hamiltonian systems (such as this one) is often analyzed by computing the Melnikov function [34]

$M=\int_{0}^{\infty}\left\{\psi_{0}\left(x_{0}, y_{0}\right), \psi_{1}\left(x_{0}, y_{0}\right)\right\} \mathrm{d} t$,

where $\{f, g\}=\partial_{x} f \partial_{y} g-\partial_{y} f \partial_{x} g$ is the Poisson bracket of functions $f$ and $g$. The streamfunction is approximated to first order as $\psi(x, y)=$ $\psi_{0}(x, y)+\epsilon \psi_{1}(x, y, t)$, where $\psi_{0}$ is the unperturbed (time-independent) portion of the streamfunction and $\psi_{1}$ is the first-order approximation to the time-periodic perturbation:

$\psi_{0}(x, y)=-\frac{a d}{\pi} \cos \left(\frac{\pi x}{d}\right) \cos \left(\frac{\pi y}{d}\right)$,

$\psi_{1}(x, y)=a \sin \left(\frac{\pi x}{d}\right) \cos \left(\frac{\pi y}{d}\right) \sin (\omega t)$,

where $a$ and $d$ are both 1, as before. In Eq. (6), $x_{0}$ and $y_{0}$ refer to coordinates of a trajectory connecting the two fixed points along an unperturbed separatrix (i.e., if $\epsilon=0$ ).

The Melnikov integral $M$ represents a first-order approximation of the variation $\Delta \psi_{0}$ of the streamfunction encountered by a tracer as it moves in the vicinity of the separatrix between adjacent vortices. It is related to the flux $F$ of tracers during one oscillation period by the relation [34]

$F=\epsilon \frac{4}{\pi}|M|$.

For a normally diffusive process, this flux can then be related to the enhanced diffusion coefficient $D^{*}$ by Fick's law: $F=D^{*} \partial \bar{c} / \partial x$, where $\bar{c}$ is the coarse-grained concentration field, smoothed to remove variations within a unit cell of the flow. Consequently, $|M|$ should be proportional to $D^{*}$.

The absolute value of the Melnikov function for this system is plotted as a function of oscillation frequency $f$ in Fig. 7a. Comparing this figure with Fig. 5, it is clear that $|M|$ does not capture the dominant frequency dependence of $D^{*}$. In particular, the two main resonance peaks from Fig. 5 are completely missing in the Melnikov analysis.
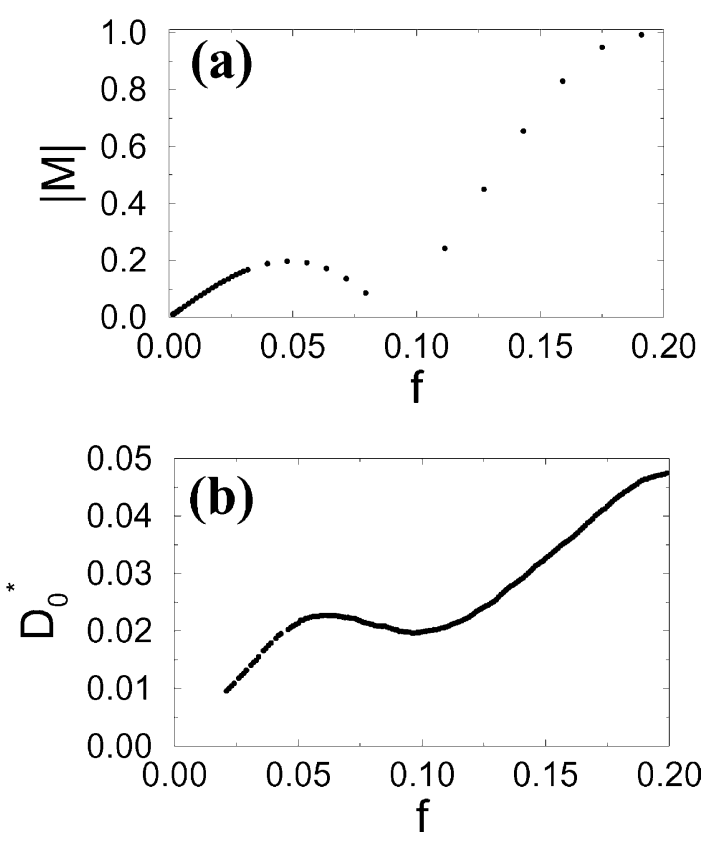

Fig. 7. Melnikov analysis: (a) plot of absolute value of Melnikov function $|M|$ versus frequency; (b) initial value of enhanced diffusion coefficient $D_{0}^{*}$, determined from the slope of $\left\langle x^{2}\right\rangle$ versus $t$ for $t<5$ for numerical data in Figs. 4-6.

The Melnikov function does not incorporate tracer motion that occurs over timescales longer than a single oscillation period. As was discussed in Section 3 in conjuction with Fig. 4, significant deviations in the transport occur only after several periods of oscillation; consequently, it is not surprising that the Melnikov analysis fails to capture the frequency dependence of the long-time behavior of $D^{*}$.

The results of these studies indicate that care must be taken when using Melnikov analysis to study transport; specifically, the presence of flights will destroy the ability of the Melnikov technique to capture long-term behavior. The Melnikov analysis, however, does capture the very short-term transport properties, as shown in Fig. 7b, which shows the short-time limit of the enhanced diffusion coefficient $D_{0}^{*}$, which is $D^{*}$ calculated for $t<5$ from the same data used to generate Fig. 5. This is further evidence that the resonance behavior in Fig. 5 is due to tracer motion that occurs over timescales much longer than a single oscillation period. 


\subsection{Lobes}

Another approach commonly used to analyze transport involves characterizing lobes $[7,25,26]$ which provide the mechanism for transport between vortices. Whereas a simple analysis of "primary" lobes suffers from the same limitations as the Melnikov analysis (i.e., capturing behavior over only the timescale of a single oscillation), a generalization based on "secondary" intersections can capture longer term behavior.

A cartoon illustrating lobe analysis is shown in Fig. 8. Two hyperbolic fixed points are shown as open circles, along with the unstable manifold of the top one (solid curve) and the stable manifold of the bottom one (dashed curve). If the $2 \mathrm{D}$ flow is time-independent (Fig. 8a), the two manifolds coincide and form a separatrix dividing two regions of the flow. In this case, transport can occur past this unperturbed separatrix only via molecular diffusion. If the flow is time-periodic (Fig. 8b and c), though, the manifolds do not coincide; rather, they become very complex structures that intersect at numerous locations between the two fixed points.

The regions bounded by the manifolds (identified by numbers - 1, 2, 3, 4 - and letters - a, b, c in Fig. 8) are the lobes. Any tracers that are contained in lobe \#1 at one instant will end up in lobe \#2 one (a)

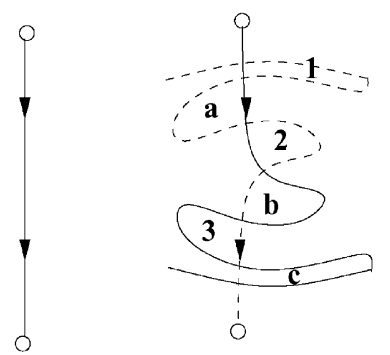

(c)

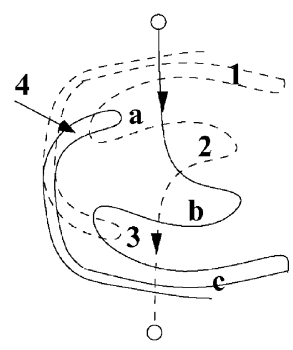

Fig. 8. Cartoon sketch of lobe analysis. The solid (dashed) curve represents the unstable (stable) manifold of the upper (lower) fixed point. The intersections of these manifolds are the lobes. (a) Unperturbed (time-independent) case. The unstable manifold of the upper fixed point coincides with the stable manifold of the lower fixed point. (b), (c) Perturbed (time-periodic) case; (b) shows only the primary lobes, whereas (c) shows a secondary intersection as well. period later, then \#3, etc. Similarly, tracers in lobe a will move to lobe $\mathrm{b}$ after one period, then lobe $\mathrm{c}$, etc. The result is that tracers to the left of the unperturbed (time-independent) separatrix - i.e., those in lobe a - cross over to the right of the unperturbed separatrix and vice versa.

This is the primary advective mechanism for mixing between vortices and for long-range transport. Since the flow is incompressible, the flux of tracers from one vortex to the next is determined by the area of the primary lobe. (This is the area of any of the numbered or lettered lobes in Fig. 8 - they must have the same area.)

The lobe picture is more complicated for a spatially periodic system, such as the oscillating vortex flow studied here (Fig. 9), particularly if the oscillation period is longer than the advective timescale $\tau_{\mathrm{adv}}$,

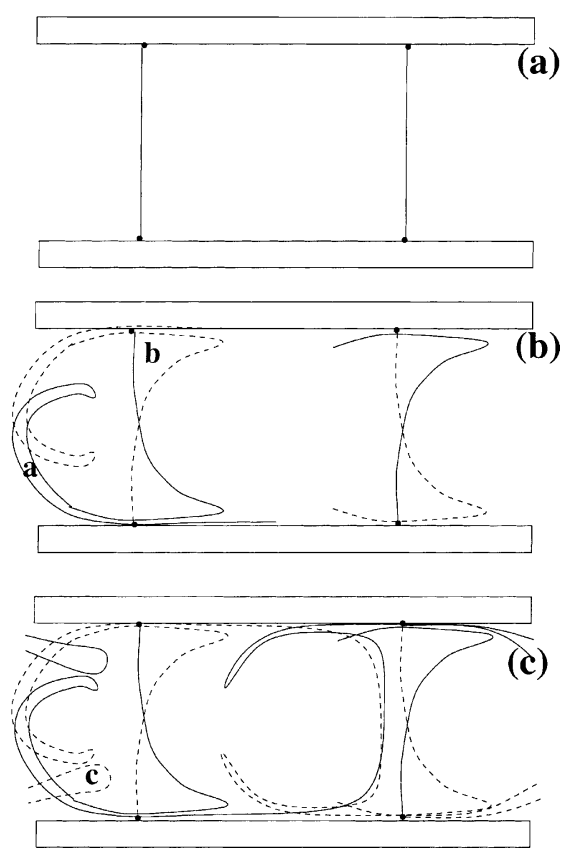

Fig. 9. Simplified sketch of lobe analysis for oscillating vortex chain. The solid (dashed) curve represents the unstable (stable) manifold of the upper (lower) fixed point. (a) Unperturbed (time-independent) case. The unstable manifolds of the upper fixed points coincide with the stable manifolds of the lower fixed points and form the separatrices between adjacent vortices. (b), (c) Perturbed (time-periodic) case; (b) shows the primary lobes responsible for the flux of impurities across a vortex boundary; (c) shows a secondary intersection (near the lower left). 
in which case there is typically significant stretching and folding of the lobes after only a couple of oscillation periods. Nevertheless, the same mechanism applies for mixing of tracers between adjacent vortices.

Previous experiments $[17,18]$ demonstrated that an analysis of the areas of the lobes (such as lobe $b$ in Fig. 9b) can successfully predict long-range transport properties of the system. Specifically, the flux determined from these lobe areas were used successfully to predict the enhanced diffusion coefficient $D^{*}$. However, this clearly is not the entire picture. As outlined in Section 4.1, any analysis that depends only on the flux between vortices during one period cannot fully explain the frequency-dependence of $D^{*}$, nor can it explain the transient superdiffusion observed in the vicinity of the resonance peaks. It is clear, then, that the previous experiments were at a frequency away from resonance peaks.

The lobe analysis can be extended to account for behavior that occurs over timescales longer than a single oscillation period. Theoretical studies [35] have indicated that secondary lobe intersections can have a significant effect on the transport properties of the system. The basic idea is illustrated in Figs. 8c and 9c. If there is overlap between different lobes, then the flux between vortices can be different from that inferred only from the area of the primary lobes. In Fig. 8c, for instance, tracers that start in lobe \#1 move into lobe \#2 one period later, then cross over the unperturbed separatrix into lobe \#3 and then lobe \#4 in successive periods. But part of lobe \#4 overlaps with lobe a, and that portion will cross back over the unperturbed separatrix to the right into lobe $\mathrm{b}$ in the following period. The net result is a reduction in the long-term flux of tracers from the right to the left.

In a periodic system - such as the one studied here - secondary intersections can result in enhancements in the flux beyond those determined from the primary lobe areas. In Fig. 9c, tracers that are in lobe a cross a separatrix into lobe $b$ after one period. After the next oscillation period, these tracers are in lobe c, which can stretch across another vortex separatrix since the velocity field repeats after two vortices. (Fig. 9 is displayed with periodic boundary conditions.) As drawn in Fig. 9c, a portion of lobe c overlaps with lobe a; tracers in that overlap region will cross over another unperturbed separatrix during the next oscillation period, enhancing the transport.

Illustrating the effects of secondary lobe intersections for this system is problematic. First, for the frequencies studied here, the lobes are extremely complicated. An idea of this complicated structure can be inferred from Fig. 2. In both of the parts of this figure, the second image from the bottom shows the tracer locations after one oscillation period, and the bottom after two oscillation periods. Although, not rigorously a plot of the manifolds, the manifolds have the same complexity as the tracer patterns outside the center vortex. As discussed above, it is necessary to look at behavior over timescales of more than a few oscillation periods (tens of periods, in fact). The manifolds after two periods are hopelessly complex; in fact, so much stretching and folding occurs in a few periods that there are dozens of different intersections.

Attempts at characterizing differences in the secondary intersections in this system near the resonance peaks have been unsuccessful due to the complexity of the manifolds for this system and to the long time scales required.

\subsection{Resonant Lévy flights and periodic orbits}

When confronted with superdiffusive transport, it is natural to ask if the trajectories can be described as Lévy flights. Qualitative evidence of flights is shown in Fig. 10, which shows plots of horizontal position versus time. The bottom curve corresponds to a frequency $f=0.095$, just outside the peak region of Fig. 5 ,

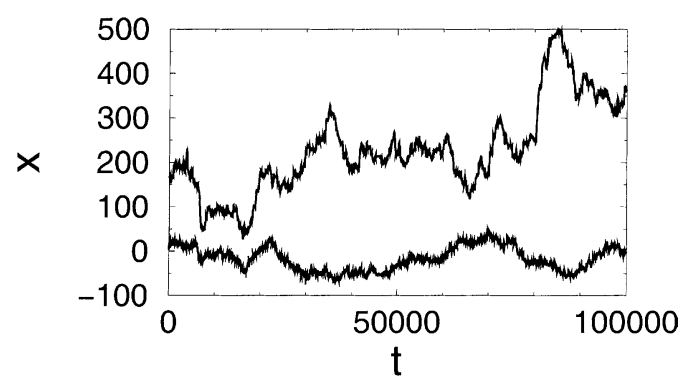

Fig. 10. Horizontal position of tracer particles versus time; $f=0.106$ (upper, dashed curve), $f=0.095$ (lower, solid curve). 

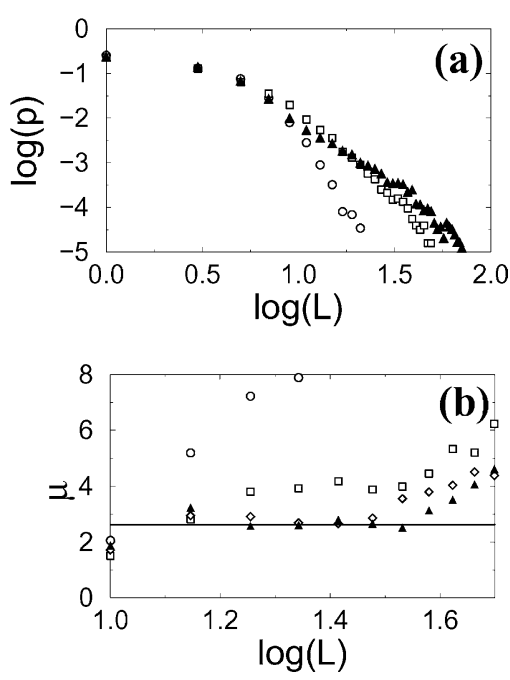

Fig. 11. Flight length PDFs; $f=0.095$ (open circles), $f=0.103$ (open squares), $f=0.105$ (open diamonds in (b) only), $f=0.106$ (filled triangles). (a) Logarithmic plots. (b) Negative of the (smoothed) slopes of the plots from (a), revealing the exponent $\mu$ as a function of length. The horizontal line corresponds to $\mu=2.6$.

whereas the top curve corresponds to $f=0.106$, close to the resonance peak. Jumps can be seen in the trajectory for $f=0.106$ (some over a hundred vortex widths), reminiscent of Lévy flights. PDFs $p(L)$ of these flights are plotted in Fig. 11. At the resonant frequency ( $f=0.106$, filled triangles), there is a significant scaling region where $p(L) \sim L^{-\mu}$ with $\mu=2.6$ up to $L=40(\log (L)=1.6),{ }^{5}$ although $p(L)$ decays more rapidly for jumps longer than 40 vortex widths. Technically, these are not Lévy flights, since the enhanced decay of $p(L)$ at large $L$ gives the flights a finite second moment. However, the power-law scaling for smaller $L$ explains the transient superdiffusive growth of the variance observed at and near this frequency. Despite concerns about the use of Eq. (3) for predicting transient behavior, this short-length value of $\mu$ is consistent with the prediction from Eq. (3) and Fig. 6 for the scaling growth of the variance: $\gamma=$ $4-\mu$.

\footnotetext{
${ }^{5}$ The slopes in Fig. 11a have been smoothed to obtain the graph of $\mu$ in Fig. 11b; consequently, the scaling region appears to be slightly smaller when plotted in Fig. $11 \mathrm{~b}$.
}

For frequencies very close to the resonant frequency ( $f=0.105$, open diamonds in Fig. 11b), $p(L)$ still scales as a power with an exponent $\mu=2.6$, although $p(L)$ deviates from this relation at a smaller length $L$ than for the resonant frequency. As the frequency deviates more and more from the resonant frequency ( $f=0.103$ and 0.095 , open squares and circles, respectively), the deviation from a power law with Lévy scaling occurs at smaller and smaller flight lengths until (outside the resonance hump) there is no appreciable power-law scaling region at all. This is consistent with the observations of transient superdiffusion with smaller and smaller duration as the frequency deviates from the resonant frequency (Fig. 6).

The fact that the tracers undergo flights with Lévy scaling for small-to-moderate lengths explains why the flux-based analyses of the previous two sections fail for this system. As is common in transport with flights, a small number of tracers undergoing very long flights can dominate the variance calculations. In general, any analysis that depends on calculation of flux for one oscillation period will fail in a system where long flights are significant (i.e., with PDFs with significant power-law regimes).

An explanation of the origin of the flights can be obtained by extending the lobe analysis, looking not just at secondary intersections, but at where the intersections occur. A secondary lobe intersection - such as that sketched in Fig. $9 \mathrm{c}$ - is a necessary condition, but not sufficient for long flights; such an intersection guarantees only that the tracer will cross into one additional vortex before its forward progress ends. For a tracer to undergo a long flight (orders of magnitude longer than a vortex width), the tracer must return to almost the exact same location in the new lobe (some number of vortices down the chain) as it was in the original lobe. In other words, there has to be a periodic orbit (modulo $2 n$ vortex widths) within the lobe. Ideally, if a tracer returned (after one oscillation period) to exactly the same location shifted by either $n \lambda$ or by $-n \lambda$, then the tracer will always find itself in a lobe to be carried across a vortex separatrix and will undergo an infinite flight. This ideal situation is never encountered; however, it is possible to get close to a resonant condition and achieve very long flights. 


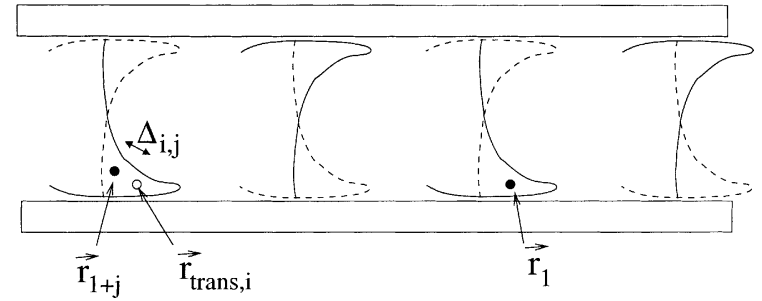

Fig. 12. Sketch illustrating calculation of translated return map. The distance $\Delta_{i, j}=\left|\vec{r}_{\text {trans }, i}-\vec{r}_{1+j}\right|$ is the separation after $j$ iterations of a tracer from its original position, translated horizontally by $i$ vortices. In the figure, $i$ is -2 (i.e., translated 2 vortex widths in the negative direction).

This approach can be quantified by calculating a translated return map, as is explained schematically in Fig. 12. The position of a tracer initially at location $\vec{r}_{1}$ is integrated numerically for $j$ periods of oscillation, after which the tracer's location $\vec{r}_{1+j}$ is compared to a horizontal translation of the original coordinate: $\vec{r}_{\text {trans }, i}=\vec{r}_{1}+i d \hat{x}$, where $d$ is the vortex width and $i$ is a non-zero integer (either positive or negative). In Fig. $12, \vec{r}_{\text {trans }, 2}$ is identified by an open circle. A distance $\Delta_{i, j}=\left|\vec{r}_{\text {trans }, i}-\vec{r}_{1+j}\right|$ is defined to quantify how close the tracer returns in $j$ periods to its original position, translated horizontally by $i$ vortices. If $\Delta=0$ for any point within a lobe, then there is a periodic orbit that can give rise to arbitrarily long flights.

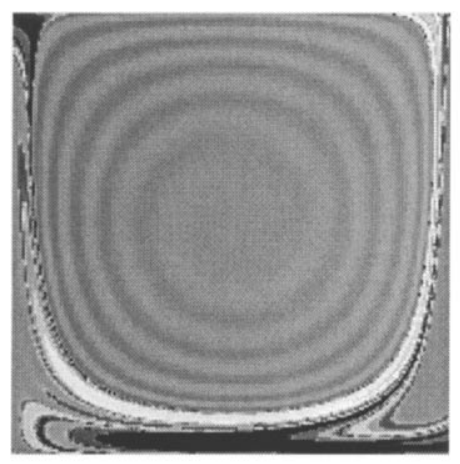

(a)
Fig. 13a shows a map of $\Delta_{4,2}$ for the resonant frequency $f=0.106$. The darkest regions where $\Delta_{4,2} \rightarrow 0-$ correspond to locations where a tracer will travel 4 vortex widths in two periods of oscillation, returning almost to the same location in the translated vortex. The brightest regions correspond to $\Delta_{4,2} \rightarrow 8$, i.e., tracers that moved four vortices in the other direction. Together, the light and dark structures in this map indicate the lobes.

Since long-range flights are associated with $\Delta \rightarrow$ 0 , it is convenient to plot $1 / \Delta$ for frequencies near the resonances. The tendency of the mixing to be dominated by long flights would then be expected to be revealed as upward peaks in the quantity $1 / \Delta$. Fig. $13 \mathrm{~b}$ shows a contour plot of $1 / \Delta_{4,2}$ (basically, the inverse of the data in Fig. 13a). The contour plot is almost completely empty, except for a couple of very narrow and very tall peaks near the bottom and center. The one closest to the middle is the highest peak, and indicates the location of a periodic orbit where a tracer will return within 0.001 of its initial location in a different vortex. Similar plots for frequencies sufficiently far from the resonances do not reveal significant peaks, indicating that none of the tracers return as close to their initial location (in a different vortex) at those frequencies.

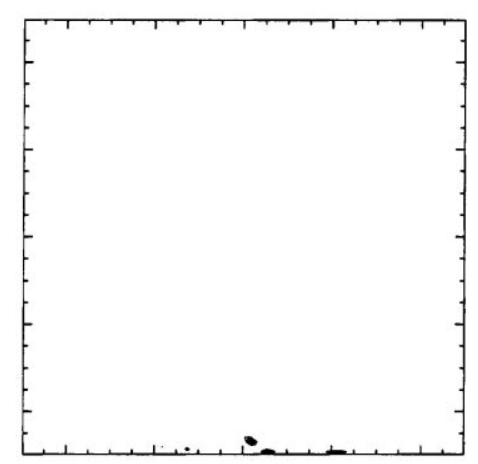

(b)

Fig. 13. Translated return maps; $f=0.106$. (a) Map of distance $\Delta_{4,2}$. The darkest regions correspond to $\Delta \rightarrow 0$; the brightest regions correspond to $\Delta \rightarrow 8$. (b) Contour plot of $1 / \Delta_{4,2}$; five equally spaced levels are shown, although they are difficult to distinguish because the peaks - located at the center near the bottom — are so sharp. The sharp peaks (especially the one just right and above the center bottom) correspond to the locations of the periodic orbits. 


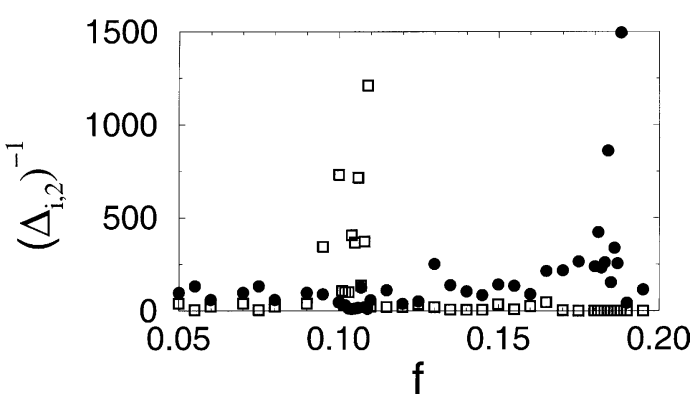

Fig. 14. Inverse of the minimum shifted distance $\Delta_{i, j}$ for tracers after $j=2$ complete periods of oscillation. A large value on this plot means that a tracer has returned to almost its exact initial condition, but shifted by either $i=2$ vortex widths (filled circles) or by $i=4$ vortex widths (open squares).

The results are summarized in Fig. 14, which shows a plot of the inverse of the minimum return distances $1 / \Delta_{ \pm 4,2}$ (open squares) and $1 / \Delta_{ \pm 2,2}$ (filled circles), corresponding to shifts of either 4 or 2 vortex widths, respectively, after two oscillation periods. There is significant scatter in the data, since the minimum distance determined numerically is very sensitive to the initial placement of the tracers. Nevertheless, it is clear that there is a strong correlation between this diagnostic and the peaks observed in the plot of $D^{*}$ versus $f$ (Fig. 5).

Note that the velocity of the flights at $f=0.106$ is roughly twice the velocity of the flights at $f=0.183$. This is apparent from the fact that the peak at $f=$ 0.183 in Fig. 14 corresponds to tracers that translate by 2 vortex widths in two oscillation periods (filled circles), whereas the peak at $f=0.106$ corresponds to tracers that translate by 4 vortex widths in the same time.

The peaks seen in Fig. 14 at the resonant frequencies are associated with formation of periodic orbits and (when stable) their associated islands, as seen in Fig. 15. In Fig. 15c, two small islands can be seen in the lower lobe regions (see Fig. 13 for the locations of the lobes), along with small thin islands along the separatrices at the sides (outside the lobes). The islands in the lobe are magnified in Fig. 15d. The islands in
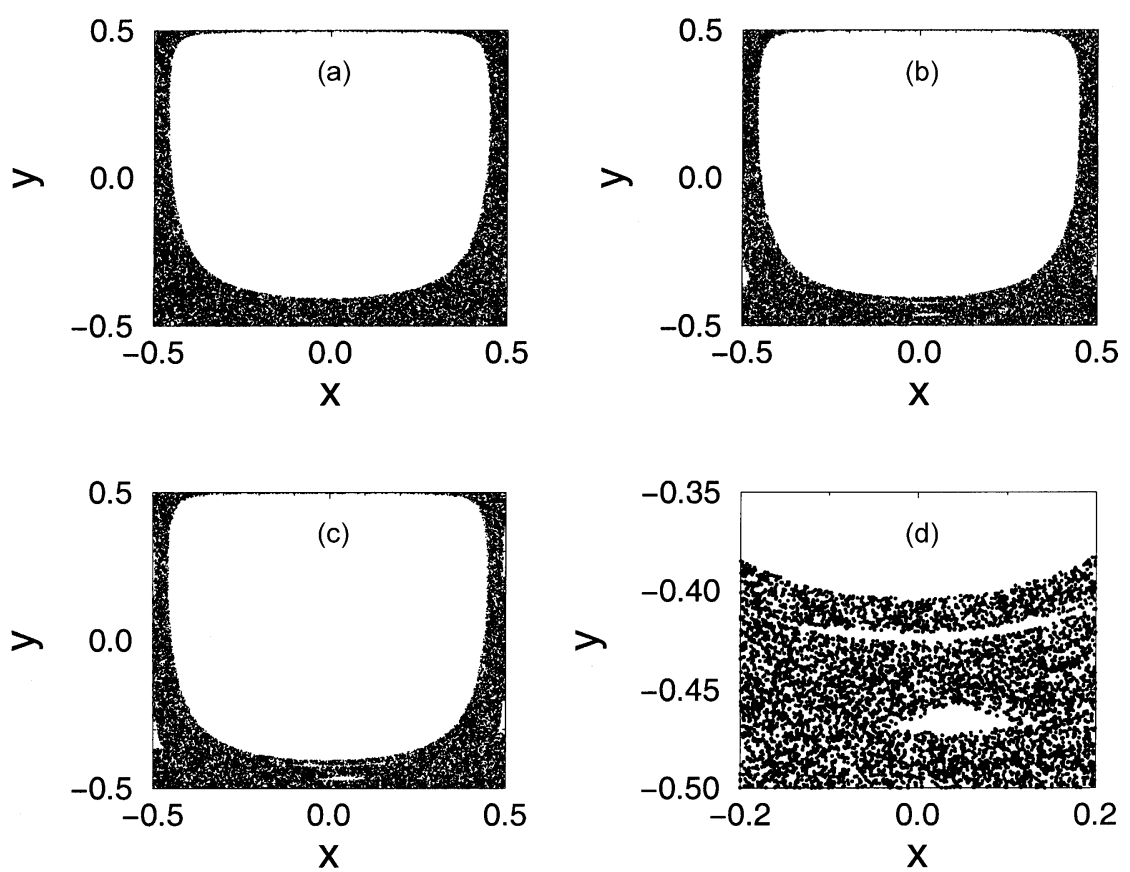

Fig. 15. Poincaré sections showing the formation of tangle islands at the resonant frequency. (a) $f=0.103$; no islands are visible. (b) $f=0.105$; very small islands are just forming near the center/bottom and along the separatrices. (c) $f=0.106$; the islands, although still small, are more developed. (d) Magnification of tangle islands from (c). 
the lobes are present only for frequencies very close to the resonant frequency: at $f=0.105$ (Fig. 15b), the islands have almost disappeared, and they are gone completely for $f=0.103$ (Fig. 15a). The islands in the lobes disappear for frequencies only slightly higher than the resonant frequency as well, although the islands along the separatrices (not in the lobes) persist for slightly larger frequencies (beyond $f=0.110$ ).

The formation of periodic orbits and islands only at certain frequencies is clearly due to resonances between a circulation time for tracers near the separatrix and the oscillation period. The presence of the "tangle islands" [20] (islands within lobes - related to accelerator modes [36]) explains the superdiffusive behavior at the resonance frequencies. But even at frequencies where the tangle islands are gone (e.g., at $f=0.103$ ), there are still truncated flights (with finite $\left\langle L^{2}\right\rangle$ ) and transient superdiffusion. This is not surprising, since at frequencies just below or just above those with islands there are still ghosts of the islands - periodic or near-periodic orbits that are just slightly unstable. The fact that these orbits are almost neutrally stable explains why a tracer could stay in the vicinity of these ghosts long enough to undergo a significant flight, but not long enough to allow for Lévy scaling for arbitrarily large flight lengths. Even though the superdiffusion is transient, these slightly unstable orbits can still result in significant enhancements in the long-term effective diffusion coefficient.

\section{Summary}

In this paper, we have demonstrated a resonant mechanism by which flights can occur in a simple, 2D, time-periodic, oscillating vortex flow with stress-free boundary conditions. Unlike Lévy flights studied in other systems, these flights are characterized by power-law PDFs only in the vicinity of certain well-defined resonant frequencies, and only the smaller length flights satisfy the Lévy (power law) scaling, with the upper cut-off decaying as the frequency of oscillation moves away from the resonant frequencies. The result is transport that is superdiffusive in the long-time limit only precisely at the resonant frequencies, as shown in previous studies [19]. Near these resonant frequencies, there is still a superdiffusive transient regime, and the long-term effective diffusion coefficient $D^{*}$ is greatly enhanced.

The flights are explained by considering translated return maps for the system. Specifically, long flights and long superdiffusive transients are characterized by tracers which, when initially in a lobe, return to almost the exact same location in a lobe of a different vortex after a finite number of oscillation periods. These periodic orbits are associated with the formation of tangle islands (islands within the lobes) at the resonant frequencies. Ghosts of these islands which are only slightly unstable at nearby frequencies result in the transient superdiffusion.

The current work illustrates some of the limitations of the traditional techniques for analyzing transport in Hamiltonian systems - Melnikov analysis and lobe dynamics. Although, these techniques work well for transport where the motion can be described on a fixed, periodic time scale, they fail to capture the (potentially dramatic) effects on transport of long-range flights.

We are currently extending this work to investigate mixing in an oscillating vortex chain with rigid (no-slip) boundary conditions. Experiments are also being conducted on the frequency dependence of enhanced diffusion coefficients. The results from these simulations and experiments will be presented in a future paper.

\section{Acknowledgements}

We are pleased to acknowledge discussions with V. Rom-Kedar, G. Zaslavsky, D. del-Castillo-Negrete, A. Vulpiani and I. Mezić. The studies are supported by NSF grants DMR-0071771 and PHY-9732158.

\section{References}

[1] H. Aref, J. Fluid Mech. 143 (1984) 1.

[2] D.V. Khakhar, H. Rising, J.M. Ottino, J. Fluid Mech. 172 (1986) 419.

[3] J. Chaiken, R. Chevray, M. Tabor, W.M. Tam, Proc. R. Soc. London Ser. A 408 (1987) 165. 
[4] J.M. Ottino, The Kinematics of Mixing: Stretching, Chaos, and Transport, Cambridge University Press, Cambridge, 1989.

[5] R.S. MacKay, J.D. Meiss, I.C. Percival, Physica D 13 (1984) 55.

[6] T.H. Solomon, J.P. Gollub, Phys. Rev. A 38 (1988) 6280.

[7] R. Camassa, S. Wiggins, Phys. Rev. A 43 (1991) 774.

[8] A.J. Majda, P.R. Kramer, Phys. Rep. 314 (1999) 237.

[9] E.W. Montroll, M.F. Shlesinger, in: J.L. Lebowitz, E.W. Montroll (Eds.), Nonequilibrium Phenomena. II. From Stochastics to Hydrodynamics, Studies in Statistical Mechanics, Vol. II, North-Holland, Amsterdam, 1984, p. 1.

[10] M.F. Shlesinger, G.M. Zaslavsky, J. Klafter, Nature 363 (1993) 31.

[11] J. Klafter, A. Blumen, M.F. Shlesinger, Phys. Rev. A 35 (1987) 3081.

[12] T.H. Solomon, E.R. Weeks, H.L. Swinney, Phys. Rev. Lett. 71 (1993) 3975.

[13] T.H. Solomon, E.R. Weeks, H.L. Swinney, Physica D 76 (1994) 70.

[14] M. Ding, T. Bountis, E. Ott, Phys. Lett. A 151 (1990) 395.

[15] Y.-C. Lai, M. Ding, C. Grebogi, R. Blümel, Phys. Rev. A 46 (1992) 4661.

[16] G.M. Zaslavsky, D. Stevens, H. Weitzner, Phys. Rev. E 48 (1993) 1683.

[17] T.H. Solomon, S. Tomas, J.L. Warner, Phys. Rev. Lett. 77 (1996) 2682.

[18] T.H. Solomon, S. Tomas, J.L. Warner, Phys. Fluids 10 (1998) 342.

[19] P. Castiglione, A. Crisanti, A. Mazzino, M. Vergassola, A. Vulpiani, J. Phys. A 31 (1998) 7197.

[20] V. Rom-Kedar, G. Zaslavsky, Chaos 9 (1999) 697.
[21] V.I. Arnold, C.R. Acad. Sci. Paris 261 (1965) 17.

[22] A.N. Yannacopoulos, I. Mezic, G. Rowlands, G.P. King, Phys. Rev. E 57 (1998) 482.

[23] J.P. Gollub, T.H. Solomon, Phys. Scripta 40 (1989) 430.

[24] E.W. Bolton, F.H. Busse, R.M. Clever, J. Fluid Mech. 164 (1986) 469.

[25] S. Wiggins, Chaotic Transport in Dynamical Systems, Springer, New York, 1992.

[26] V. Rom-Kedar, S. Wiggins, Transport in two-dimensional maps: concepts, examples, and a comparison of the theory of Rom-Kedar and Wiggins with the Markov model of MacKay, Meiss, Ott, and Percival, Physica D 51 (1991) 248.

[27] M.F. Shlesinger, J. Statist. Phys. 10 (1974) 421.

[28] X.-J. Wang, Phys. Rev. A 45 (1992) 8407.

[29] J. Klafter, G. Zumofen, Phys. Rev. E 49 (1994) 4873.

[30] E.R. Weeks, H.L. Swinney, Phys. Rev. E 57 (1998) 4915.

[31] S. Benkadda, S. Kassibrakis, R. White, G.M. Zaslavsky, Phys. Rev. E 59 (1999) 3716.

[32] Y. Pomeau, A. Pumir, W.R. Young, C.R. Acad. Sci. 306 (1988) 741.

[33] O. Cardoso, P. Tabeling, Europhys. Lett. 7 (1988) 225.

[34] D. del-Castillo-Negrete, Phys. Fluids 10 (1998) 576.

[35] V. Rom-Kedar, Chaos 5 (1995) 385.

[36] A.J. Lichtenberg, M.A. Lieberman, Regular and Chaotic Dynamics, 2nd Edition, Springer, New York, 1992.

[37] M.A. Fogleman, M.J. Fawcett, T.H. Solomon, Phys. Rev. E 63 (2001) 020101(R).

[38] W. Young, A. Pumir, Y. Pomeau, Phys. Fluids A 1 (1989) 462.

[39] O. Cardoso, P. Tabeling, Eur. J. Mech. B 8 (1989) 459. 